\title{
High Resolution Density Map as the Visualization of a Larger Time Scale Molecular Dynamics
}

\author{
P. KARBOWNICZEK \\ Institute of Physics, Cracow University of Technology, Podchorążych 1, 30-084 Kraków, Poland \\ (Received October 29, 2014; revised version March 18, 2015; in final form March 25, 2015)
}

\begin{abstract}
A method of visualization of two-dimensional systems of particles in a large time scale molecular dynamics with application to the domain reorientation process in 2D Lennard-Jones system is presented. The described processing is general and can be used in comparing experimental results of atomic force microscopy with the results calculated using a computer as well as for predicting new phenomena. Domain reorientation process in 2D Lennard-Jones system approaching equilibrium is presented and confronted with the Fourier analysis of the data obtained for different initial parameters and at different states of aggregation. New border domain features accompanying this process are discussed.
\end{abstract}

DOI: $10.12693 /$ APhysPolA.127.1644

PACS: 61.20.-p, 64.70.dm, 61.20.Ja

\section{Introduction}

Imaging of systems in molecular dynamics (MD) simulations is as old concept as the MD method itself. The most standard way to visualize the system is to plot circles in the atoms positions. More sophisticated method is to look at the traces of the atoms to have a first insight into their mobility aspects. Next step in visualization is to create density maps. This idea requires averaging over many configurations that exist in the time proximity. It can be done for short time averages to observe transient effects or the structure in the local densities. Introducing high resolution density maps (HRDM) with large averaging time with pixel color suppression/adjustment to fit quality requirements allows a new possibility in analysis. Results of this method turn out to be perfectly suitable for the high resolution fast Fourier transform (FFT) analysis, which is the most reliable tool in studying symmetry of the structures, hence, helping in judging of the stability or the metastable regions in the solid state.

Contrary to the earlier attempts of MD imaging, it lies close to the idea of the atomic force microscopy (AFM) pictures. AFM imaging [1] is a powerful method which within the last 25 years has been successfully used to study the surfaces with the resolution ranging from $10^{-10} \mathrm{~m}$ till $10^{-4} \mathrm{~m}$. In principle, AFM is a mechanical instrument whose performance is limited by the speed of the tip moving over the substrate, hence resulting in a relatively slow imaging rate. Conventional AFM typically requires more than one minute to perform a topographic image, depending on image area. As a consequence the AFM registers an average of many states present at the molecular level. Moreover, different parts of the image are not taken at the same time. If the scanning starts, for instance, at the left side and proceeds to the right side, the right part of the image is, in fact, taken much later than the part of the starting point. This can be meaningless if the sample features are stable on the time level of taking AFM picture, but cannot be used to observe fast changes due to, for example, molecular arrangements.
The idea of collecting HRDM like the one used in AFM images is exactly at the root of the present paper simulation idea. It is also aimed at creating a tool allowing comparison of the AFM results and MD simulations, since it follows the natural state of the particles. A two-dimensional case with the interaction among particles modeled by the Lennard-Jones potential has been chosen. The particular parameters used correspond to argon, for which the Lennard-Jones potential is known to provide a very good description. This potential, however, has a drawback of not being able to describe properly solids, where more sophisticated or ab initio models are in use. The standard way of performing MD consists of two parts. In the first, an attempt to equilibrate the system is undertaken, in the second, much shorter than the previous one and called the production run, a number of observables are calculated. The type of the phase obtained can be judged from studying the correlation functions character. In 2D case these are radial distribution function $g(r)[2]$ and radial bond orientational correlation functions $g_{4}(r)$ and $g_{6}(r)$ [3] that allow to find the type of arrangement. These properties have easily interpretable features if the system is well equilibrated. They become, however, insufficient if the system is dynamically changing. By this we mean that they can be time dependent and its properties can be blurred by the time changes. The drawbacks of the above methods can be overcome using density maps of the whole particle system collected at many subsequent instants. The use of the two-dimensional geometry and the systems bounded by a rectangular box (as presented further) provides an additional asset to the visualization.

\section{High resolution density maps}

A high resolution density map is created here as a matrix $M_{m n}$. If the particle system is limited by the rectangle of the volume $V=a b$, then every element of the density matrix $M$ of the size $m \times n$ corresponds to the volume of $a b / m n$, which establishes the resolution of the computer experiment. The matrix $M_{m n}$ is constructed 
as follows. (a) Create matrix $M_{m n}$, (b) fill in each element of the matrix with 0 , (c) at the time of collecting data, for each $i$-th particle located in $(x, y)$ find its corresponding location in the matrix as $(1+($ int $)(m x / a)$, $1+($ int $)(n y / b))$, and increment the value of the matrix element by $1,(d)$ repeat step (b) for selected time moments until the simulation reaches the desired time. The high resolution photographic type of the image is obtained then using the pixel intensity suppression and application of the full grey scale range. $M$ matrix is a $2 \mathrm{D}$ histogram, whose largest values are in locations, where the particles spend the most of their time. For good visualization or for the purposes to study the effects of longer simulations, the statistics needs a substantial amount of the computer calculation time. Even if the building of the matrix $M$ has been started at the beginning of the simulations, their current image is being dominated by the last positions of the particles. This method can be also called "one instant snapshot rule", since all the parts of the image come from the evaluation due to the same time conditions and the building of the histogram is stopped at the same instant for each part of the system. Matrix generated is transformed into a gray-scale photographic image. For the purpose of the symmetry analysis the FFT has been used. This method (the density map plus the FFT analysis) will be not successful in distinguishing the liquid from the gas, since these phases have almost homogeneous density maps, however, it will differentiate the liquid from the solid phase, allow for the characterization of the structures at the liquid-solid interface and for the identification of different solid configurations. Also, indirectly through the degree of blurriness, it gives insight into their dynamics.

\section{Example}

The HRDM method was used for a two-dimensional system of argon particles interacting via truncated and shifted Lennard-Jones potential

$$
U(r)= \begin{cases}4 \epsilon\left[(\sigma / r)^{12}-(\sigma / r)^{6}\right], & \\ -4 \epsilon\left[\left(\sigma / r_{\mathrm{c}}\right)^{12}-\left(\sigma / r_{\mathrm{c}}\right)^{6}\right], & r<r_{\mathrm{c}} \\ 0, & r \geq r_{\mathrm{c}}\end{cases}
$$

where $\epsilon$ is the depth of the potential well, $\sigma$ is the distance at which the inter-particle potential is zero; $r$ is the inter-atomic distance, $r_{\mathrm{c}}$ is the cut-off radius. Initially the atoms were arranged on $2 \mathrm{D}$ simple square lattice with periodic boundary conditions. The total number of the particles used here is equal to 900 . All computations were performed in the reduced units with the time step equal to $\Delta t=0.002$. The parameters of argon $\left(M=0.03994 \mathrm{~kg} / \mathrm{mol}, T=119.8 \mathrm{~K}, \rho=1680 \mathrm{~kg} / \mathrm{m}^{3}\right)$ have been transformed to the reduced units in which $t^{*}=1$ is a reduced time corresponding to real time equal to $2.18 \mathrm{ps}$. Note that this unit is so small that it is unattainable in real experimental conditions. The system was initially simulated for $10^{5}$ time steps. Under NVE conditions, the system finally evolved to certain conditions of the pressure $P$ and temperature $T$. Starting from this moment, calculations for the histogram were performed every 10 time steps. Different histograms for $10^{4}$, $10^{5}$ and $10^{7}$ time steps (corresponding to $44 \mathrm{ps}, 440 \mathrm{ps}$ and $44 \mathrm{~ns}$ ) for different initial total energy and density were calculated. Therefore, the results presented later for the various times represent different, but sufficient enough, number of averaged configurations taken into account. The properties of the HRDMs depend largely on the time of the simulation, however, smaller statistics can affect the sharpness of the image only by a small extent. The size of the matrix $M$ was chosen as 500 by 500 , which gives 250000 points of the histogram. Pixel intensity suppression has been used to expose the details of the image. The resulting ratio of the particles number to the number of the matrix $M$ points is $3.6 \times 10^{-3}$. Small number here is indispensable for the quality of the histogram.

In the subsequent pictures HRDMs and their FFT transforms are presented for liquid (Fig. 1), liquid-solid interface (Fig. 2) and solid state (Fig. 3). The parameters of these particular states examples have been chosen from the density-temperature phase diagram [4]. Whereas for small times the HRDM of the liquid phase has well distinguishable features with the FFT showing a concentric ring without spots (Fig. 1a-c), for longer times it reaches the state for which the HRDM is uniform (structureless).

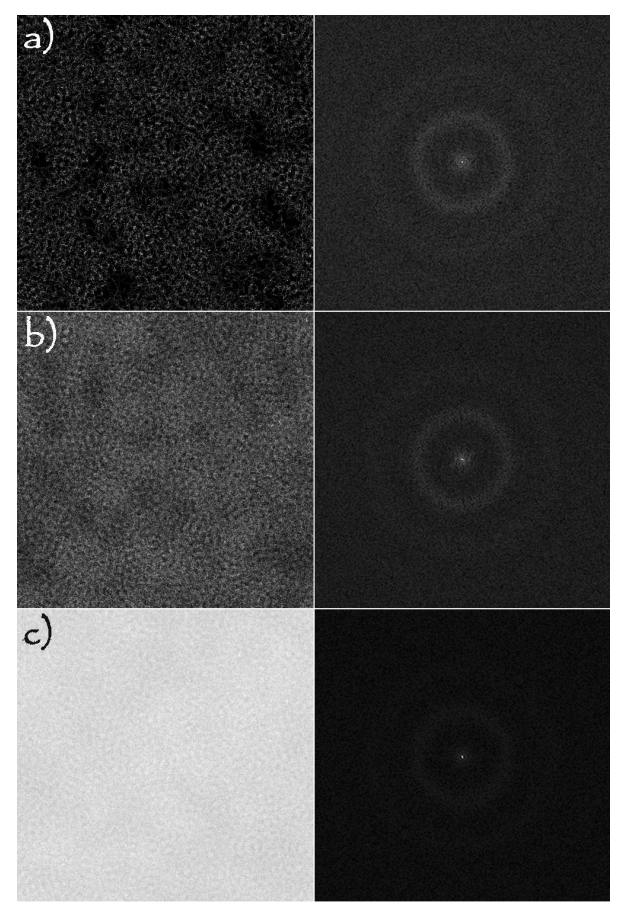

Fig. 1. HRDMs for (a) $10^{4}$, (b) $10^{5}$, (c) $10^{7}$ time steps corresponding to $44 \mathrm{ps}, 440 \mathrm{ps}$ and $44 \mathrm{~ns}$. The density is equal to $\rho=0.50$, when the calculated temperature $T=1.0$ (liquid). The corresponding FFT images are shown on the right side.

In the transition region between the solid and fluid (Fig. 2) one observes polycrystalline domains at shorter times (part (a)), which gradually disappear while simulating, forming hexagonal type structure (part (b) 


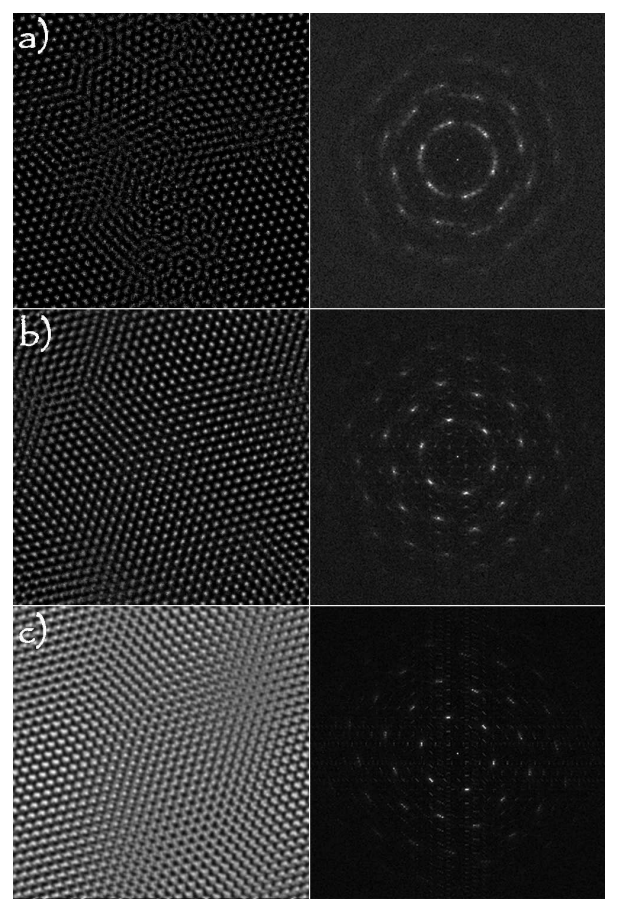

Fig. 2. HRDMs for (a) $10^{4}$, (b) $10^{5}$, (c) $10^{7}$ time steps corresponding to $44 \mathrm{ps}, 440 \mathrm{ps}$ and $44 \mathrm{~ns}$. The density is equal to $\rho=0.95$, when the calculated temperature $T=1.0$. The corresponding FFT images are shown on the right side.

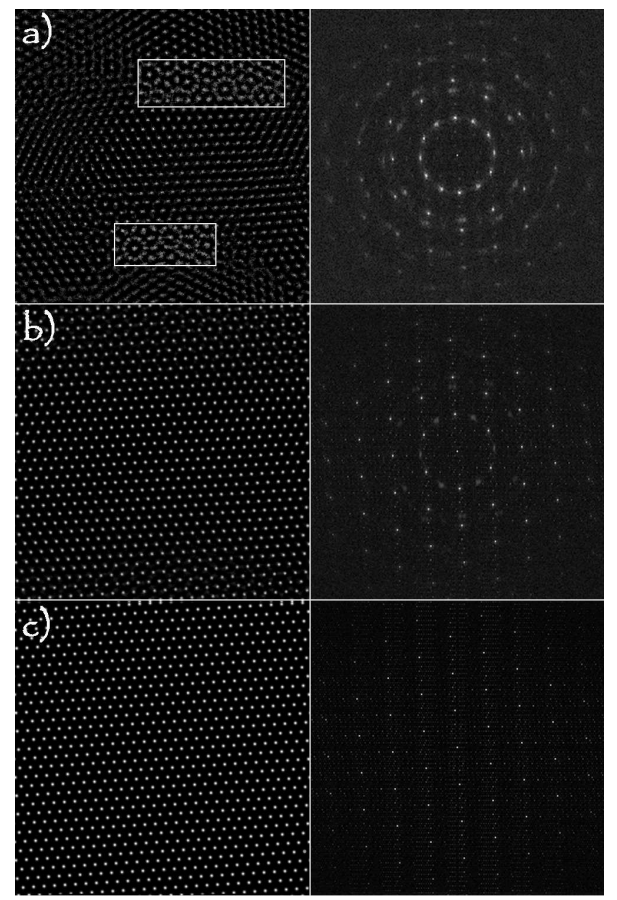

Fig. 3. HRDMs for (a) $10^{4}$, (b) $10^{5}$, (c) $10^{7}$ time steps corresponding to $44 \mathrm{ps}, 440 \mathrm{ps}$ and $44 \mathrm{~ns}$. The density is equal to $\rho=1.00$, when the calculated temperature $T=1.0$ (solid). The corresponding FFT images are shown on the right side. and (c)) with peculiar FFT image which at much later times takes on the form of hexagonal structure with slight disclinations (blurred long areas where the direction of the raw of particles is slightly though noticeably changed). The absence of long range spatial order is apparent from the FFT image. This effect would not be so clearly visible in the short time HRDMs.

The number of the polycrystalline domains decreases with increasing density; the process is quite sensitive to the value of the density: a small change in density results in very large changes in the structure. A well defined crystalline structure is visible in the area of solid (Fig. 3). From the snapshots presented here another important question can be drawn, which concerns the problem of decision whether the system has reached equilibrium level in the simulations. Looking at Fig. 3 for the time steps corresponding to 44 ps (part (a)) one observes the domain or polycrystalline-like structure which disappears already in part (b) configuration for the time of $440 \mathrm{ps}$. The hexagonal patterns are confirmed in parts (b) and (c) by the Fourier images. Comparison of parts (c) of Figs. 2 and 3 shows that probability of domain reorganization, visible only in a short time scale, is expected to be higher for lower densities. In polycrystalline system one observes new features at the border areas. These are the particles that are practically immobile but surrounded by very mobile neighbors (see highlighted areas in Fig. 3). Enhanced mobility of these particles at the interfacial areas or domain borders can be justified by enhanced blurriness of their images. Once detected such a group of the particles can be put under more detailed examination.

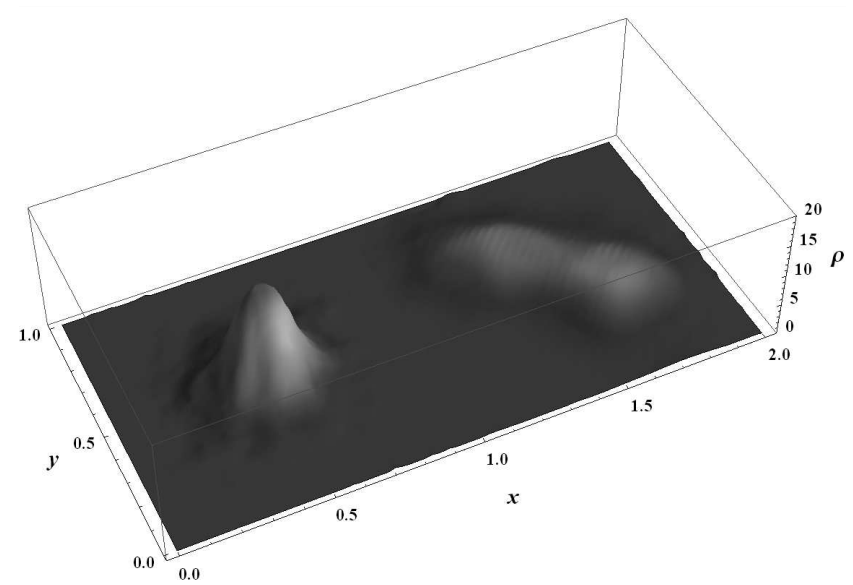

Fig. 4. Comparison of the density profiles of the normal particles with Gaussian-like distribution and more mobile particles at the polycrystallinity borders.

Figure 4 presents the density profiles of the normal particles and their more mobile neighbors, the effect discovered by the using of the HRDM method. This nonequilibrium feature will strongly influence diffusion properties at these areas. Also the chains of such mobile particles groups may be responsible for the easiness of the domain reorientations. Targeting such specific mobility 
situations is an important problem in nanotechnology applications, microfluidic mixing and interfacial phenomena.

\section{Summary}

As a summary future importance of this method should be emphasized. It should be noticed that the described method allows to observe new effects that otherwise may not be possible to detect (like the presented enhanced mobility at the polycrystallinity borders) with the presentation of the single particle configuration (Fig. 5) or by the macroscopic or local density averaging (with results similar to fluid case in Fig. 1). The method can

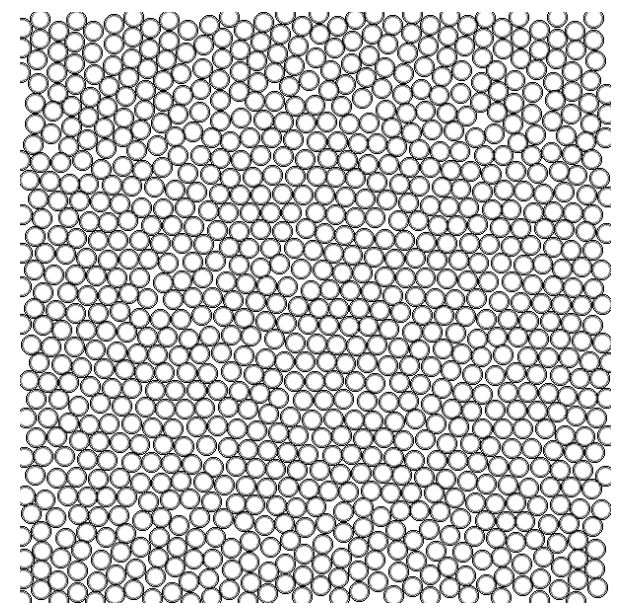

Fig. 5. The exemplary configuration of the particles corresponding to the simulation with the parameters from the part (a) of Fig. 3.

be successfully used to determine the size and the number of domains and to determine time evolution of the domain patterns. Solid state can be easily distinguished from liquid and gas. HRDM textures carry also some indication of the diffusion coefficient. The crystal structure will have a small diffusion constant, whereas the fuzzy features indicate larger diffusivity. The resulting histogram images are similar to the pictures created in the AFM experiments. If the system studied by AFM is not changing over scanning time then there is even a chance to directly compare the both methods outcome. Applications of the HRDM method may help to cast a new light on the controversial problems. Until now, for instance, there exists ambiguity as far as the existence of the hexatic phase in 2D systems (also the Lennard-Jones system) (see for instance [3] and references therein) is concerned. According to [3] such a phase occurs between solid and liquid state. The hexatic phase described by the Kosterlitz, Thouless, Halperin, Nelson, and Young (KTHNY) theory [5-8], is subject to the size effects and stabilizes only when the simulation system is large [3]. Its presence is indicated by the algebraic decay of the bond orientational order parameter as a consequence of the disclinations. The HRDM seems to be a promising tool to study these effects, however, much more simulation work is needed here. An undeniable advantage of the presented HRDM method is also the full time control of creating maps and the fact that the different parts of the image are being observed at the same time. In the case of AFM, even though the ultra-high speed instruments are capable to catch 1000 (or more) frames per second [9, 10], so being capable to cover the distance of nanometers in nanoseconds, still different parts of the picture are taken at a different instant, providing averages of dynamical changes at the molecular time scale at incommensurate time conditions. Because of the "one instant snapshot" rule the HRDM method can be regarded then as a complementary to AFM source of information.

\section{Acknowledgments}

The author is thankful to P. Zieliński and A. Chrzanowska, Institute of Physics, Cracow University of Technology for their comments and suggestions for improving the quality of the paper.

\section{References}

[1] G. Binnig, C.F. Quate, Ch. Gerber, Phys. Rev. Lett. 56, 930 (1986).

[2] A.A. Khan, Phys. Rev. 134, A367 (1964).

[3] K. Chen, T. Kaplan, M. Mostoller, Phys. Rev. Lett. 74, 4019 (1995).

[4] J.A. Barker, D. Henderson, F.F. Abraham, Physica A 106, 226 (1981).

[5] J.M. Kosterlitz, D.J. Thouless, J. Phys. C: Solid State Phys. 6, 1181 (1973).

[6] B.I. Halperin, D.R. Nelson, Phys. Rev. Lett. 41, 121 (1978).

[7] D.R. Nelson, B.I. Halperin, Phys. Rev. B 19, 2457 (1979).

[8] A.P. Young, Phys. Rev. B 19, 1855 (1979).

[9] L.M. Picco, L. Bozec, A. Ulcinas, D.J. Engledew, M. Antognozzi, M.A. Horton, M.J. Miles, Nanotechnology 18, 044030 (2007).

[10] J.A. Vicary, M.J. Miles, Nanotechnology 20, 095302 (2009). 